\title{
Extrinsic allergic alveolitis induced by the yeast Debaryomyces Hansenii
}

\author{
Y. Yamamoto*,\#, S. Osanai*, S. Fujiuchi\#, K. Yamazaki*, H. Nakano*, Y. Ohsaki*, K. Kikuchi*
}

Extrinsic allergic alveolitis induced by the yeast Debaryomyces Hansenii. Y. Yamamoto, S. Osanai, S. Fujiuchi, K. Yamazaki, H. Nakano, Y. Ohsaki, K. Kikuchi. (C) ERS Journals Ltd 2002.

ABSTRACT: A 65-yr-old female developed cough, fever and dyspnoea following repeated exposure to a home ultrasonic humidifier. High-resolution computed tomography showed ground-glass opacity in both lung fields. Arterial blood gas analysis gave an oxygen tension of $8.38 \mathrm{kPa}$ (63 Torr). Pulmonary function testing revealed restrictive ventilatory impairment with a reduction in the diffusing capacity. The diagnosis of extrinsic allergic alveolitis (EAA) was confirmed by radiographic findings, pathological evidence of alveolitis and reproductive development by a provocation test to the humidifier water. The yeast Debaryomyces Hansenii was the only microorganism cultured from the water of the humidifier. The double diffusion precipitating test and lymphocyte proliferative response was positive for an extract of $D$. Hansenii, providing evidence to incriminate this fungus. This is the first described case of EAA caused by $D$. Hansenii.

Eur Respir J 2002; 20: 1351-1353.
*First Dept of Medicine, Asahikawa
Medical College and ${ }^{\#}$ Dept of Respira-
tory Medicine, National Douhoku
Hospital, Hanasaki, Asahikawa, Japan.

Correspondence: Y. Yamamoto, First Dept of Medicine, Asahikawa Medical College, 2-1-1-1, Midorigaoka-Higashi, Asahikawa 078-8510, Japan.

Fax: 81166682449

E-mail: yasushiy@asahikawa.hosp.go.jp

Keywords: Air conditioning, alveolitis, extrinsic allergic, inhalation exposure, Debaryomyces Hansenii

Received: April 132002

Accepted after revision: April 292002
Extrinsic allergic alveolitis (EAA) is an immunologically mediated lung disease resulting from exposure to inhaled organic dusts [1]. Fungi represent a large source of antigens capable of causing EAA, including Alternaria, Aspergillus, and Penicillium [2]. The yeast Debaryomyces Hansenii appears as a further dominant species in indoor air samplings [3], whereas it has not been described as an aero-antigen for EAA. The first case of EAA induced by D. Hansenii, which contaminated a home ultrasonic humidifier, is reported here.

\section{Case presentation}

A 65-year-old female was admitted to Asahikawa Medical College Hospital because of nonproductive cough, dyspnoea and fever. She had no suggestive history of atopy and had never smoked. No pets were kept in her house. She had a low-grade fever together with evidence of sinus tachycardia, tachypnoea and bibasilar fine crackles. A chest radiograph revealed an increase in lung density predominantly on the left lower fields. High-resolution chest computed tomography demonstrated ground-glass opacity and fine nodules (fig. 1).

Arterial blood gas showed a $\mathrm{pH}$ of 7.45 , carbon dioxide tension of $5.43 \mathrm{kPa}(41 \mathrm{mmHg})$, and oxygen tension of $8.38 \mathrm{kPa}(63 \mathrm{mmHg})$. A pulmonary function test revealed a restrictive pattern associated with mild airway obstruction and a reduction in diffusing capacity. The white blood cell count (WBC) was
$15,500 \cdot \mathrm{mm}^{-3}$ with $80 \%$ neutrophils and $2 \%$ eosinophils. The erythrocyte sedimentation rate was $51 \mathrm{~mm} \cdot \mathrm{h}^{-1}$. The total immunoglobulin (Ig)E was normal. Cultures for microorganisms from her sputum grew normal flora.

Following treatment with antibiotics, her symptoms and objective findings improved within 1 week. On the twelfth day of hospitalisation, she left the hospital to stay overnight at her home. Approximately $6 \mathrm{~h}$ after returning home, her illness was reproduced. Following her return to hospital on the next day, fever and cough were spontaneously resolved without any specific treatments. A series of recurrent episodes with a selflimiting course led to a presumptive diagnosis of EAA. On close questioning, she recalled that an ultrasonic home humidifier was employed in her living room prior to the first onset of her illness. She had used it every day except during hospitalisation periods, without cleaning of the water reservoir.

To confirm a reproductive development of EAA by the humidifier, a provocation test was performed. With the humidifier operating at her bedside, the patient was exposed to the humidifier mist for $1 \mathrm{~h}$ in the closed room (fig. 2).

She developed cough, fever and dyspnoea $4 \mathrm{~h}$ after provocation. A reduction in the carbon monoxide diffusing capacity of the lung, forced vital capacity and forced expiratory volume in one second occurred maximally from $4-8 \mathrm{~h}$ after challenge. These values returned towards baseline levels at $24 \mathrm{~h}$. Progression of hypoxia and increases in WBC were also observed, which persisted for $48 \mathrm{~h}$. Bronchoscopic examination 


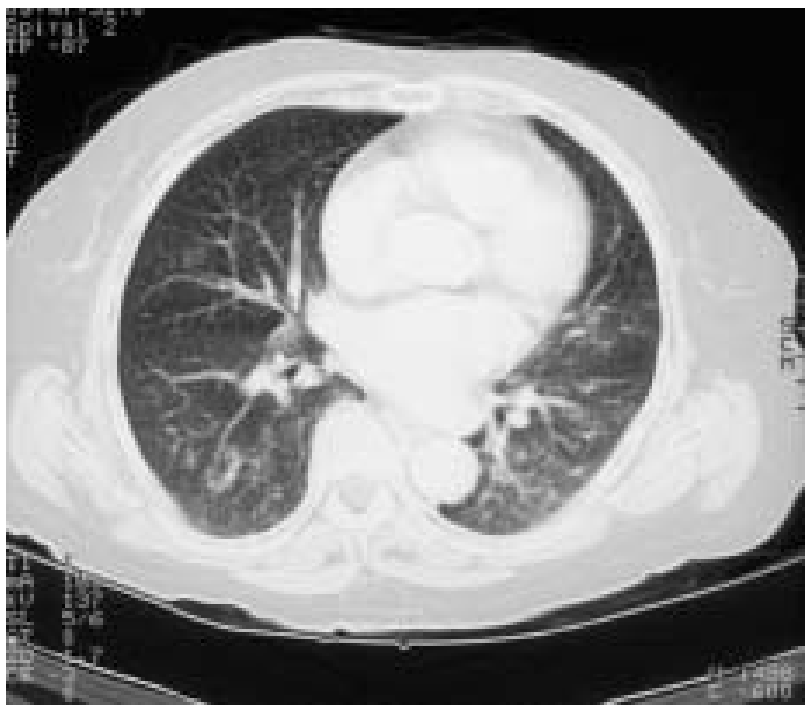

Fig. 1.- High-resolution chest computed tomography scan showing ground-glass opacity and fine nodules predominantly on the lower lung fields.

was performed at $96 \mathrm{~h}$ after the provocation test. Transbronchial lung biopsy specimens revealed alveolitis with lymphocytic infiltration. Bronchoalveolar lavage (BAL) fluid cell count showed alveolar macrophages of $62 \%$, lymphocytes of $26 \%$ and neutrophils of $12 \%$

Samplings obtained from the humidifier water in the reservoir were cultured with potato dextrose and heart-infusion agar. The yeast $D$. Hansenii was isolated from the culture plate colonies. No other fungus and bacteria could be identified. Purified

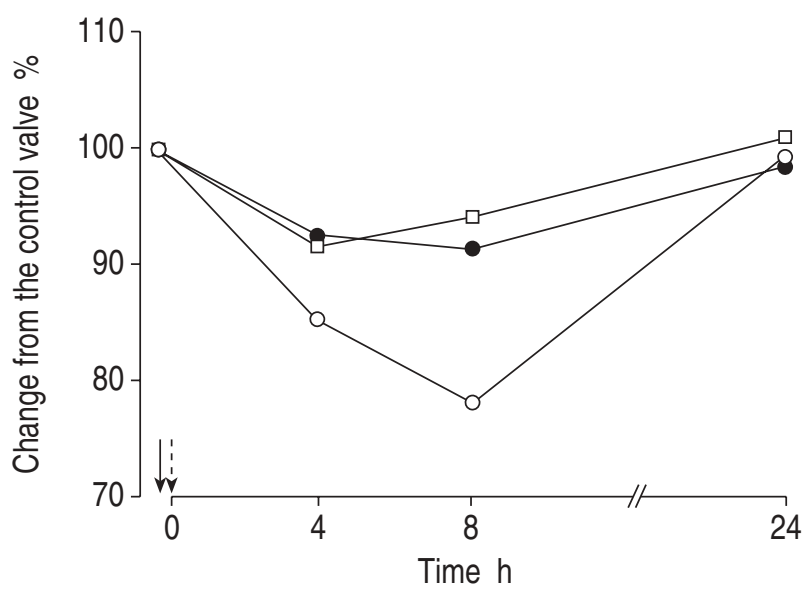

Fig. 2.-Result of bronchial provocation test by inhalation of humidifier water. The carbon monoxide diffusing capacity of the lung (open circles), forced expiratory volume in one second (closed circles), and forced vital capacity (open squares) decreased maximally at $4-8 \mathrm{~h}$ after provocation. Elevation in body temperature, leucocytosis and corresponding hypoxia were also observed. Solid arrow: control; dashed arrow: provocation. $0 \mathrm{~h}$ : body temperature $\left(\mathrm{BT}^{\circ} \mathrm{C}\right): 36.1, \mathrm{WBC} \cdot \mathrm{mm}^{-3}: 7,940$, oxygen tension in arterial blood $\left(\mathrm{Pa}_{\mathrm{a}} \mathrm{O}_{2}\right): 9.86 \mathrm{kPa}(74.1 \mathrm{Torr}) ; 4 \mathrm{~h}: \mathrm{BT}^{\circ} \mathrm{C}: 37.5$, white blood cell count (WBC· $\left.\mathrm{mm}^{-3}\right): 7,520, \mathrm{~Pa}_{\mathrm{a}} \mathrm{O}: 8.09 \mathrm{kPa}(60.8$ Torr); $8 \mathrm{~h}: \mathrm{BT}^{\circ} \mathrm{C}$ : $38.1, \mathrm{WBC} \cdot \mathrm{mm}^{-3}: 19,830, \mathrm{~Pa}_{\mathrm{a}} \mathrm{O}_{2}: 8.37 \mathrm{kPa}(62.9$ Torr); 24 h: $\mathrm{BT}^{\circ} \mathrm{C}: 38.8, \mathrm{WBC} \cdot \mathrm{mm}^{-3}: 16,200, \mathrm{~Pa}_{\mathrm{O}}: 8.63 \mathrm{kPa}$ (64.9 Torr). polysaccharide antigen was extracted from the colonies to detect a serum-precipitating antibody. Ouchterlony double diffusion tests showed striking precipitin bands in response to both the humidifier water and $D$. Hansenii extract. In contrast, her serum did not show any precipitin reactions against the following antigens: Thermoactinomyces vulgaris, Micropolyspora faeni, Cephalosporium, Aspergillus, and Pigeon droppings. A lymphocyte proliferative response test for peripheral blood cells was also positive for the $D$. Hansenii extract.

The diagnosis of EAA was confirmed by: the presence of repeated symptoms after exposure, antibody detection in serum, radiographical findings, pathological evidence of alveolitis, and positive inhalation challenge test [4]. She was discharged after removal of the humidifier from her home. Her symptoms improved dramatically, and there was no repeated exacerbation.

\section{Discussion}

Humidifying or ventilatory systems in buildings are occasionally contaminated with thermophilic microorganisms, resulting in the development of EAA [2]. Examination of the recent literature on ventilationassociated EAA revealed an ultrasonic humidifier to be the major origin of antigenic agents [5-8]. However, there have been few reports successfully identifying the antigens responsible. Similarly to previous reports, the present case was not associated with thermophilic microorganisms, because the ultrasonic humidifier generated aerosols without heating. This suggests that other potent antigen(s) can induce EAA associated with the use of an ultrasonic humidifier. A wide variety of specific IgG antibodies are detected in the patients with humidifier lung, indicating that no single microorganism can be implicated in the aetiology [9]. To the best of the authors knowledge the present report is the first well-documented case of Debaryomyces-induced EAA.

D. Hansenii is characterised by high tolerability to osmotic stress; thus, it can survive in an aquatic environment [10]. The optimal growth temperature ranges from $25-30^{\circ} \mathrm{C}$ [11]. In view of these biological features of Debaryomyces, the environment in the water reservoir of the ultrasonic humidifier seemed to be suitable for its growth. Although air sampling from the patient's house was not performed, it is speculated that maintaining the humidifier in a mouldy environment might lead to gross contamination with Debaryomyces, resulting in extensive exposure to the present patient.

The genus Debaryomyces is normally a nonpathogenic yeast classified to the Ascomycetes [12]. D. Hansenii has rarely been isolated from humans, with the only case of bone infection being demonstrated in an immunocompetent host [11]. Therefore, the question can be raised as to why D. Hansenii is capable of inducing sensitisation and development of EAA. The genus Saccharomyces, belonging to the Ascomycetes, is known to produce allergic respiratory symptoms due to the intrinsic enzyme enolase [13]. 
The most likely explanation is that Debaryomyces may also release some allergens such as intrinsic enzymes, polysaccharides and mycotoxin on the patient's distal airway and alveolus [14].

Most patients with EAA have lymphocytes that respond to antigen-induced blastogenesis in their peripheral blood [15] and BAL fluid [16]. This fact indicates that cell-mediated immunity may play an important role in the pathogenesis of EAA. Debaryomyces extract also induced a proliferative effect on the patient's peripheral lymphocytes, suggesting that Debaryomyces could initiate a cell-mediated hypersensitivity response. Furthermore, the presence of serum-precipitating antibody against Debaryomyces was a supportive finding for antigen identification $[4,17]$. Finally, the reproduction by a provocation test, with an exposure to aerosol originating from the causative humidifier was confirmed. Although an inhalation challenge by Debaryomyces extract was not undertaken, due to unexpected risks, the authors believe that these findings provide evidence to incriminate Debaryomyces.

This case highlights Debaryomyces Hansenii as a newly recognised antigen. To conclude, an investigation into Debaryomyces should be considered in cases where there is a failure to detect the common antigens in extrinsic allergic alveolitis.

\section{References}

1. Costabel U. The alveolitis of hypersensitivity pneumonitis. Eur Respir J 1988; 1: 5-9.

2. Fink JN. Hypersensitivity pneumonitis. J Allergy Clin Immunol 1984; 74: 1-9.

3. Carmo-Sousa L. Distribution of Yeasts in Nature. In: Rose AH and Harrison JS, eds. The Yeasts, Biology of Yeasts. London, Academic Press, 1969; pp. 79-105.

4. Schuyler M, Cormier Y. The diagnosis of hypersensitivity pneumonitis. Chest 1997; 111: 534-536.

5. Shiue ST, Scherzer HH, DeGraff JAC, Cole SR. Hypersensitivity pneumonitis associated with the use of ultrasonic humidifiers. N Y State J Med 1990; 90: 263-265.

6. Volpe BT, Sulavik SB, Tran P, Apter A. Hypersensitivity pneumonitis associated with a portable home humidifier. Conn Med 1991; 55: 571-573.

7. Kane GC, Marx JJ, Prince DS. Hypersensitivity pneumonitis secondary to Klebsiella oxytoca. A new cause of humidifier lung. Chest 1993; 104: 627-629.

8. Suda T, Sato A, Ida M, Gemma H, Hayakawa H, Chida K. Hypersensitivity pneumonitis associated with home ultrasonic humidifiers. Chest 1995; 107: 711-717.

9. Baur X, Richter G, Pethran A, Czuppon AB, Schwaiblmair M. Increased prevalence of IgGinduced sensitization and hypersensitivity pneumonitis (humidifier lung) in nonsmokers exposed to aerosols of a contaminated air conditioner. Respiration 1992; 59: 211-214.

10. Larsson C, Morales C, Gustafsson L, Adler L. Osmoregulation of the salt-tolerant yeast Debaryomyces hansenii grown in a chemostat at different salinities. J Bacteriol 1990; 172: 1769-1774.

11. Wong B, Kiehn TE, Edwards F, et al. Bone infection caused by Debaryomyces hansenii in a normal host: a case report. J Clin Microbiol 1982; 16: 545-548.

12. Warren NG, Hazen KC. Candida, Cryptococcus, and other yeasts of medical importance. In: Murray PR, eds. Manual of Clinical Microbiology. 7th edn. Washington, D.C., ASM PRESS, 1998; pp. 1184 1199.

13. Baldo BA, Baker RS. Inhalant allergies to fungi: reactions to Baker's yeast (Saccharomyces cerevisiae) and identification of Baker's yeast enolase as an important allergen. Int Archs Allergy Appl Immun 1988; 86: 201-208.

14. Baldo BA. Allergenic crossreactivity of fungi with emphasis on yeasts: strategies for further study (editorial). Clin Exp Allergy 1995; 25: 488-492.

15. Moore VL, Pedersen GM, Hauser WC, Fink JN. A study of lung lavage materials in patients with hypersensitivity pneumonitis: In vitro response to mitogen and antigen in pigeon breeders' disease. J Allergy Clin Immunol 1980; 65: 365-370.

16. Johnson MA, Nemeth A, Condez A, Clarke SW, Poulter LW. Cell-mediated immunity in pigeon breeders' lung: the effect of removal from antigen exposure. Eur Respir J 1989; 2: 445-450.

17. Burrell R, Rylander R. A critical review of the role of precipitins in hypersensitivity pneumonitis. Eur $J$ Respir Dis 1981; 62: 332-343. 